\title{
Flow cytometric analysis of the effect of dithiothreitol on leukocyte surface markers
}

\author{
D. Loppow*******, M. Böttcher**, G. Gercken***, H. Magnussen*, R.A. Jörres*
}

\begin{abstract}
Flow cytometric analysis of the effect of dithiothreitol on leucocyte surface markers. $D$. Loppow, M. Böttcher, G. Gercken, H. Magnussen, R.A. Jörres. (C)ERS Journals Ltd 2000. ABSTRACT: Pretreatment with dithiothreitol (DTT) is necessary to dissolve mucus in samples of induced sputum prior to analysis. However, DTT may affect cell surface markers which are essential for lymphocyte subtyping. Therefore, the aim of this study was to evaluate the effect of DTT on an appropriate panel of surface markers. Peripheral blood leukocytes were used because these cells, in contrast to sputum cells, could be obtained without DTT treatment.

Peripheral blood from healthy donors was incubated with either DTT according to standard sputum procedures or phosphate-buffered saline (PBS), washed and incubated with fluorochrome-labelled antibodies. After lysis of erythrocytes, analysis was performed using a calibrated flow cytometer. Leukocyte populations were identified by their light scattering properties. For analysis, fluorescence intensity was compared between DTT- and PBS-treated samples.

After treatment with DTT, fluorescence intensity was significantly increased in CD16-positive granulocytes; it was reduced in CD2-positive lymphocytes, CD45positive lymphocytes and CD14-positive monocytes $(p \leq 0.001)$. These changes occurred in all samples. The fluorescence intensity of CD3-, CD4-, CD8-, CD19-, CD56- and histocompatibility leukocyte antigen DR-positive lymphocytes was not altered by DTT. However, there were statistically significant $(p<0.001)$, although small, changes in the percentages of leukocytes.

The present data demonstrate that, although dithiothreitol as used in sputum analysis affects some surface markers of peripheral blood leukocytes, comparability between samples concerning lymphocyte surface markers is preserved. Therefore, it is suggested that treatment of sputum samples with dithiothreitol does not invalidate the immunocytochemical analysis of lymphocytes.
\end{abstract}

Eur Respir J 2000; 16: 324-329.

The method of induced sputum is widely used as a noninvasive procedure for obtaining biological samples from the airways $[1,2]$ in order to determine their cellular and biochemical composition in relation to airway diseases [3, 4]. As sputum cells are embedded in airway secretions, the material has to be liquified in order to prepare singlecell suspensions for flow cytometry. This can be achieved by incubation with the potent reducing agent dithiothreitol (DTT, 2,3-dihydroxybutane-1,4-dithiol) [5]. Therefore, DTT is widely used in sputum processing [6-9]. However, owing to its reducing properties, DTT could affect the three-dimensional structure of proteins, which is maintained by disulphide bonds, thereby leading to changes in the availability and integrity of epitopes which could hamper immunological detection procedures [10].

Indeed, the data which are available indicate effects of DTT or dithioerythritol (DTE) on surface markers assessed by flow cytometry on eosinophils or neutrophils even if cell viability remains unchanged [11-13]. Data regarding markers that are essential for lymphocyte subtyping have been published in abstract form [14]. Recent data have shown improved cell and sputum supernatant inflammatory mediator recovery when using DTE [15]. As lymphocytes are important players in airway diseases, the
*Krankenhaus Grosshansdorf, Zentrum für Pneumologie und Thoraxchirurgie, Grosshansdorf, **Labor Dr. Kramer und Kollegen, Geesthacht, and ***Universität Hamburg, Institut für Biochemie und Lebensmittelchemie, Abteilung für Biochemie und Molekularbiologie, Hamburg, Germany.

Correspondence: D. Loppow

Krankenhaus Grosshansdorf

Zentrum für Pneumologie und Thoraxchirurgie

D-22927 Grosshansdorf

Germany

Fax: 494102692295

Keywords: Dithiothreitol

flow cytometry

induced sputum

peripheral blood leukocytes

Received: June 131999

Accepted after revision April 172000

This study was supported by Landesversicherungsanstalt (LVA), Freie und Hansestadt Hamburg, Hamburg, Germany, and Labor Dr. Kramer und Kollegen, Geesthacht, Germany. study of these cells in induced sputum deserves particular attention $[10,16,17]$. Therefore, in the present study a detailed analysis of the effects of DTT on the appropriate surface markers was performed, and careful calibration using fluorescent beads included to improve the identification of those changes in cellular distribution that were due to mere reductions in fluorescence intensity. This appears to be particularly important in the analysis of lymphocyte subpopulations, because it supports the validation of data by the computation of check sums [17, 18].

Therefore, the aim of the present study was to evaluate the effect of DTT on the detection of a specific panel of leukocyte surface markers. As it was important to study the effect of DTT in cells which had not been previously treated with this compound, it was decided to investigate peripheral blood leukocytes as a model for induced sputum cells.

\section{Material and methods}

\section{Subjects}

Peripheral blood was obtained from 15 volunteers (10 male, five female; age $20-45$ yrs) who showed a normal 
white blood cell count and were judged healthy on a clinical basis. Blood was taken by venous puncture in the morning.

\section{Processing}

Samples of ethylenediamine tetra-acetic acid anticoagulated whole blood $(100 \mu \mathrm{L})$ were incubated with either $250 \mu \mathrm{L}$ Sputolysin $(\mathrm{R})(0.1 \%$ or $6.5 \mathrm{mM}$ dithiothreitol in $100 \mathrm{mM}$ phosphate buffer, $\mathrm{pH}$ 7.0; Calbiochem, Bad Soden, Germany) according to a standard sputum procedure (final concentration $0.07 \%$ DTT) $[19,20]$ or 250 $\mu \mathrm{L}$ phosphate-buffered saline (PBS) for $30 \mathrm{~min}$ at $37^{\circ} \mathrm{C}$. Afterwards, samples were washed twice with $4 \mathrm{~mL}$ PBS and centrifuged for $5 \mathrm{~min}$ at $300 \times \mathrm{g}$. After removing the supernatant, cells were incubated for $10 \mathrm{~min}$ at room temperature $\left(25^{\circ} \mathrm{C}\right)$ with different amounts of fluorescence-labelled antibodies (table 1) according to the manufacturers' instructions. After lysis of erythrocytes with Immuno-

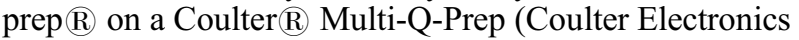
$\mathrm{GmbH}$, Krefeld, Germany), flow cytometric measurements were performed under standardized conditions using a four-colour Coulter $§$ EPICS $₫$ XL-MCL equipped with one 488-nm argon laser. All measurements were performed in duplicate to assess intraassay variability. On average (minimum), 8,698 $(4,122)$ granulocytes were counted for CD16, 1,526 (497) monocytes for CD14 and $4,850(1,337)$ lymphocytes for all other markers.

\section{Analysis}

Leukocyte populations were identified by their lightscattering properties using logarithmic sideward scatter versus linear forward scatter (fig. 1). This approach was chosen instead of the combination of CD45 expression and sideward scatter in order to maintain the same gating strategy for all surface markers, independently of potential alterations in CD45 expression. The population of

Table 1. - Antibodies and target cells used

\begin{tabular}{|c|c|c|c|}
\hline $\begin{array}{l}\text { Antibody } \\
\text { conjugate }\end{array}$ & Clone & $\begin{array}{l}\text { Target as used in } \\
\text { this study }\end{array}$ & Manufacturer \\
\hline CD14-PE & RMO22 & Monocytes & Immunotech* \\
\hline CD16-PE & $3 \mathrm{G} 8$ & Granulocytes & Immunotech* \\
\hline CD19-FITC & SJ 25-C1 & B-lymphocytes & Caltag $^{+}$ \\
\hline CD2-FITC & $39 \mathrm{C} 1.5$ & T-lymphocytes & Immunotech* \\
\hline CD3-FITC & UCHT1 & T-lymphocytes & Immunotech* \\
\hline CD4-PE & 13B8.2 & $\begin{array}{l}\text { Helper/inducer } \\
\text { T-lymphocytes }\end{array}$ & Immunotech* \\
\hline CD45-FITC & J.33 & $\begin{array}{l}\text { Leukocytes } \\
\text { (lymphocytes) }\end{array}$ & Immunotech* \\
\hline CD56-PE & B159 & NK cells & Immunotech* \\
\hline CD8-PE & B9.11 & $\begin{array}{c}\text { Cytotoxic/suppressor } \\
\text { T-lymphocytes }\end{array}$ & Immunotech* \\
\hline $\begin{array}{l}\text { HLA-DR- } \\
\text { FITC }\end{array}$ & $\mathrm{I} 3 \mathrm{O}$ & $\begin{array}{l}\text { Activated } \\
\text { lymphocytes }\end{array}$ & Coulter* \\
\hline
\end{tabular}

*: via Coulter-Immunotech, Hamburg, Germany; ${ }^{+}$: via medac, Hamburg, Germany. FITC: fluorescein isothiocyanate (fluorescence detector (FL) 1); PE: phycoerythrin (FL2); HLA: histocompatibility leukocyte antigen; NK: natural killer.

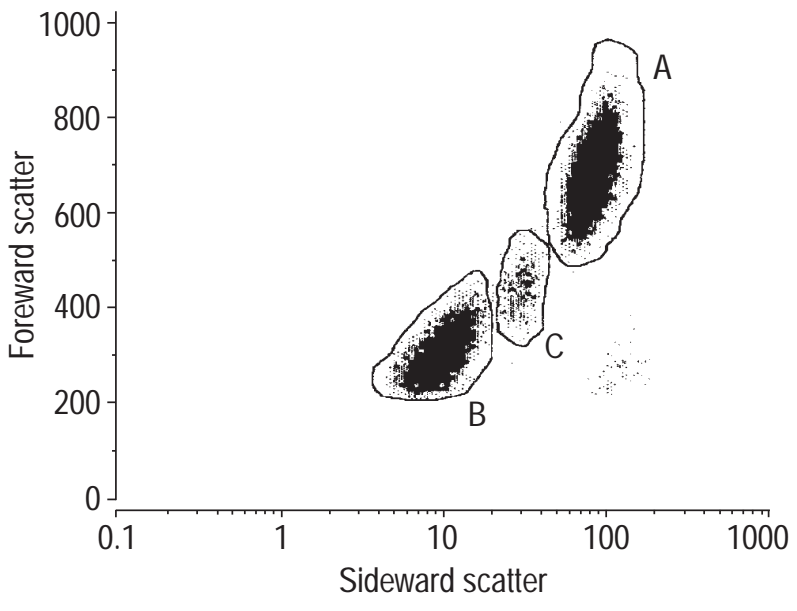

Fig. 1. - Identification of peripheral blood leukocyte population by their light scattering properties. Foreward scatter (linear) is related to cell size, sideward scatter (logarithmic) to granularity. Small mononuclear lymphocytes are shown in gate $\mathrm{B}$, monocytes in gate $\mathrm{C}$ and polymorphonuclear granulocytes in gate A.

monocytes, as identified morphologically (fig. 1, gate C), was selected for the determination of CD14, that of granulocytes (fig. 1, gate A) for CD16 and that of lymphocytes (fig. 1, gate B) for all other surface antigens studied (table 1). The numbers of blood samples studied for each antibody are given in table 2 , the variation in numbers arising from different availability of donors; it was not due to technical problems.

The flow cytometer was calibrated using DAKO-FluoroSpheres (DAKO Diagnostika GmbH, Hamburg, Germany), which consisted of blank beads and calibration beads. The latter comprised five bead populations of the same particle size labelled with different amounts of fluorochrome. After excitation by a $488 \mathrm{~nm}$ argon laser, the beads emitted signals of different fluorescense intensity which appeared in all fluorescence detectors (FL) 1-4 of the flow cytometer. The calibration-beads had been calibrated against soluble fluorochromes such as fluorescein isothiocyanate (FL1), phycoerythrin (PE; FL2), or a PE/ cyan-5 tandem conjugate (FL4) by the manufacturer. For each fluorescence colour, a calibration function was constructed in order to express signal intensity (mean channel) as the number of molecules of equivalent soluble fluorochrome (MESF). The signal of the blank beads was used to determine the level of electronic noise and the limit of detection. This standardized procedure accounted for variations in the performance of the equipment and ensured that results showed optimal reproducibility.

\section{Statistical analysis}

Throughout the analysis, means of duplicate samples were used. For the comparison of cell differentials, the individual means of the 15 subjects were taken. Cell percentages and MESF were expressed as mean \pm SEM. Changes in fluorescence intensity caused by DTT were expressed as mean \pm SD percentage differences between the DTT and PBS values relative to the PBS values. The paired t-test was used to compare DTT and PBS treatment. 
Table 2. - Intraclass correlation coefficients percentages of antibody-positive cells and fluorescence intensity in dithiothreitol (DTT)- and phosphate-buffered saline (PBS)-treated duplicate samples

\begin{tabular}{|c|c|c|c|c|c|c|c|c|c|c|}
\hline \multirow{3}{*}{ Cell types } & \multirow{3}{*}{ Ancestor } & \multirow{3}{*}{$\begin{array}{c}\text { Samples } \\
\text { n }\end{array}$} & \multicolumn{4}{|c|}{ Antibody-positive cells \% } & \multicolumn{4}{|c|}{ Fluorescence intensity MESF } \\
\hline & & & \multicolumn{2}{|c|}{$\mathrm{Ri}$} & \multirow[t]{2}{*}{ PBS } & \multirow[t]{2}{*}{ DTT } & \multicolumn{2}{|c|}{$\mathrm{Ri}$} & \multirow[t]{2}{*}{ PBS } & \multirow[t]{2}{*}{ DTT } \\
\hline & & & PBS & DTT & & & PBS & DTT & & \\
\hline Monocyte & Leukocyte & 13 & 0.92 & 0.89 & $7.55 \pm 0$ & $9.68 \pm 0$ & - & - & - & - \\
\hline Granulocyte & Leukocyte & 15 & 0.94 & 0.99 & $61.71 \pm 1.87$ & $64.41 \pm 1.9$ & - & - & - & - \\
\hline Lymphocyte & Leukocyte & 15 & 0.99 & 0.98 & $32.75 \pm 1.21$ & $29.32 \pm 1.55^{* *}$ & - & - & - & - \\
\hline CD14-pos & Monocyte & 13 & 0.92 & $0.17^{+}$ & $76.14 \pm 2.61$ & $79.10 \pm 1.54$ & 0.97 & 0.82 & $185896 \pm 6547$ & $172263 \pm 5607 * *$ \\
\hline CD16-pos & Granulocyte & 15 & 0.96 & 0.99 & $93.26 \pm 1.01$ & $94.46 \pm 0.99 * *$ & 0.92 & 0.91 & $365269 \pm 22470$ & $418443 \pm 24517 * *$ \\
\hline CD19-pos & Lymp & 10 & 0.99 & 0.90 & $10.32 \pm 1.20$ & $9.94 \pm 1.08$ & 0.88 & 0.87 & $22176 \pm 568$ & $21874 \pm 564$ \\
\hline CD2-pos & Lymphocyte & 13 & $0.47^{+}$ & 0.98 & $76.72 \pm 1.85$ & $73.00 \pm 2.04 *$ & 0.99 & 0.87 & $28938 \pm 775$ & $16387 \pm 348^{* *}$ \\
\hline CD3-pos & Lymphocyte & 10 & 0.99 & 1.00 & $70.82 \pm 2.50$ & $66.49 \pm 3.07 *$ & 0.95 & 0.97 & $97063 \pm 3295$ & $95965 \pm 3160$ \\
\hline CD4-pos & Lymphocyte & 10 & 0.99 & 0.73 & $41.51 \pm 1.90$ & $36.97 \pm 2.42 *$ & 0.82 & 0.88 & $48154 \pm 2755$ & $46898 \pm 2853$ \\
\hline CD45-pos & Lymphocyte & 12 & 0.89 & 0.93 & $96.46 \pm 0.35$ & $94.96 \pm 0.55 *$ & 0.98 & 0.97 & $149545 \pm 2319$ & $147558 \pm 2317 * *$ \\
\hline CD56-pos & Lymphocyte & 14 & 0.96 & 0.98 & $13.52 \pm 1.48$ & $14.61 \pm 1.72 *$ & 0.72 & 0.83 & $8441 \pm 284$ & $8583 \pm 350$ \\
\hline CD8-p & Lymp & 10 & 0.98 & 0.99 & $24.49 \pm 1.90$ & $23.45 \pm 1.99 *$ & 0.96 & 0.97 & $87452 \pm 3229$ & $89764 \pm 3176$ \\
\hline HLA-DR-pos & Lymphocyte & 12 & 0.69 & 0.88 & $13.40 \pm 0.60$ & $15.06 \pm 0.63^{*}$ & 0.95 & 0.94 & $148021 \pm 8717$ & $148432 \pm 8570$ \\
\hline
\end{tabular}

Data are presented as absolute values or as mean \pm SEM. ${ }^{+}$: due to outliers (see Results section); MESF: molecules of equivalent soluble fluorochromes; Ancestor: reference cell population; Ri: intraclass correlation coefficient; pos: positive; HLA: histocompatibility leukocyte antigen. $*$ : $\mathrm{p}<0.05 ; * * \mathrm{p}<0.01$.

Statistical significance was assumed at a first kind error of $\mathrm{p}<0.05$. Intra-assay reproducibility was derived from the duplicate samples by one-way analysis of variance using the intraclass correlation coefficient $(\mathrm{Ri})$. In a similar manner, the effect of DTT versus PBS was expressed in terms of $\mathrm{Ri}$.

\section{Results}

As compared to PBS, treatment with DTT caused statistically significant changes in standard cell differentials. After DTT treatment, the flow cytometer showed, on average, $2.1 \%$ more monocytes, $2.7 \%$ more granulocytes and $3.4 \%$ less lymphocytes (table 2 ; all $\mathrm{p}<0.001$ ). Corresponding $\mathrm{Ri}$ indicated high reproducibility of differential cell counts (table 2). However, there were two outliers in the case of CD14-positive monocytes (after DTT) and one outlier in the case of CD2-positive lymphocytes (after PBS).

The percentages of antibody-positive cells showed statistically significant differences (all $\mathrm{p}<0.05)$ between samples treated with PBS or DTT (table 2). After DTT treatment, the percentages of CD16-positive granulocytes, and of CD56- and histocompatibility leukocyte antigen (HLA)-DR- positive lymphocytes were increased by $1.2,1.1$ and $1.7 \%$, respectively; those of CD2-, CD3-, CD4-, CD45- and CD8-positive lymphocytes were reduced by $3.7,4.3,4.5,1.5$ and $1.1 \%$, respectively. The differences in CD14-positive monocytes (3.0\%) and CD19 -positive lymphocytes $(-0.4 \%)$ were not statistically significant. The reproducibility of duplicate samples ( $\mathrm{Ri})$ ranged 0.69-1.0, except for CD14-positive monocytes after DTT and CD2-positive lymphocytes after PBS, where Ri were $<0.5$ (table 2). Effects of DTT in relation to PBS were also visible in terms of low Ri (table 3), particularly for CD14, CD2, CD45 and HLA-DR.
Regarding fluorescence intensity, treatment with DTT led to a statistically significant (all $\mathrm{p} \leq 0.001$ ) increase in CD16-positive granulocytes, and to a reduction in CD2positive lymphocytes, CD45-positive lymphocytes and CD14-positive monocytes (table 2). No statistically significant changes occurred in CD3, CD4, CD8, CD19, CD56 and HLA-DR. Differences between duplicate samples showed Ri of $>0.7$ (table 2). Figure 2 illustrates the mean effect of DTT on fluorescence intensity. A comparison of DTT- and PBS-treated samples in terms of $\mathrm{Ri}$ is given in table 3 , demonstrating a marked effect on CD2.

\section{Discussion}

The present data demonstrate that, for a number of surface markers, DTT alters the fluorescence intensity of immunostained leukocytes as detectable by flow cytometry.

Table 3. - Intraclass correlation coefficients for percentages and fluorescence intensities between dithiothreitoland phosphate-buffered saline-treated samples.

\begin{tabular}{lccr}
\hline & & \multicolumn{2}{c}{$\mathrm{Ri}$} \\
\cline { 3 - 4 } Cell type & Ancestor & Percentage & FI \\
\hline CD14-positive & Monocyte & 0.58 & 0.77 \\
CD16-positive & Granulocyte & 0.91 & 0.82 \\
CD19-positive & Lymphocyte & 0.95 & 0.90 \\
CD2-positive & Lymphocyte & 0.54 & -0.84 \\
CD3-positive & Lymphocyte & 0.77 & 0.97 \\
CD4-positive & Lymphocyte & 0.70 & 0.83 \\
CD45-positive & Lymphocyte & 0.40 & 0.96 \\
CD56-positive & Lymphocyte & 0.96 & 0.81 \\
CD8-positive & Lymphocyte & 0.97 & 0.91 \\
HLA-DR-positive & Lymphocyte & 0.39 & 0.97 \\
\hline
\end{tabular}

Ri: intraclass correlation coefficient; Ancestor: reference cell population; FI: fluorescence intensity; HLA: histocompatibility leukocyte antigen. 


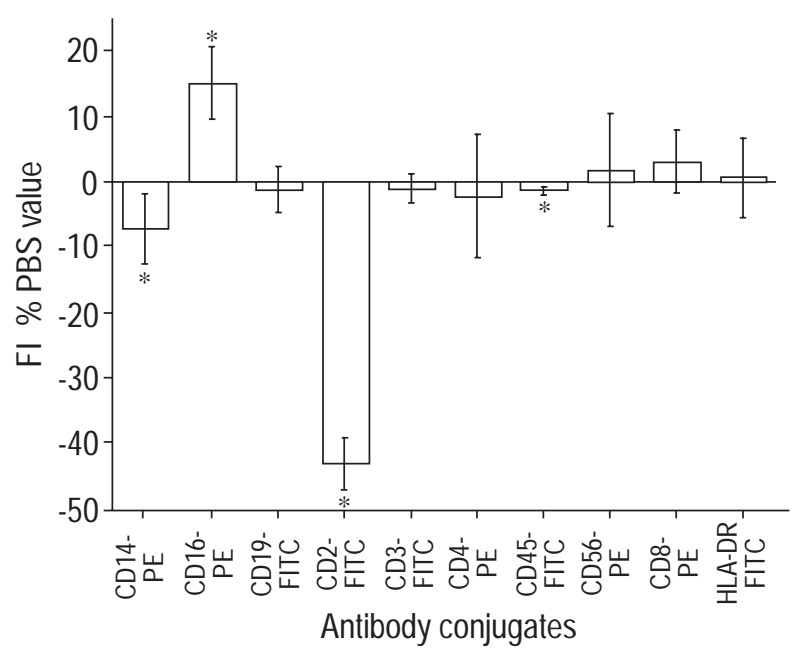

Fig. 2. - Effects of dithiothreitol (DTT) on flurescence intensity (FI). Changes in FI caused by DTT were expressed as mean \pm SD percentage differences between the DTT and phosphate-buffered saline (PBS) values relative to the PBS values. Samples were analysed in duplicate and numbers of subjects were as in table 2. PE: phycoerythrin; FITC: fluorescein isothiocyanate; HLA: histocompatibilty leukocyte antigen. *: $\mathrm{p}<0.05$ versus $\mathrm{PBS}$.

However, most changes were small and well within the range of variability of measurements. Furthermore, all studied cell populations remained well above the detection limit.

The present study was undertaken to elucidate the potential effect of DTT in sputum analysis. Sputum cells are embedded in a matrix of airway secretions produced by mucosal epithelial cells and submucosal glands [21]. The major component of this are mucins or mucus glycoproteins, which comprise up to $1-3 \%$ of sputum wet weight $[22,23]$. These components are primarily responsible for the viscosity, elasticity and adhesive capacity of mucus [24]. In order to analyse sputum cells, it is necessary to extract them from their matrix. This can be achieved by destruction of the macromolecular structure of mucins. As this is maintained by disulphide bonds, cleavage of these bonds is one approach to dissolving sputum samples. Pretreatment of sputum samples by appropriate reducing agents such as DTT is most effective and corresponding cytospin preparations yield results which are more reproducible than, for example, sputum smears [25].

The effectiveness of DTT arises from the fact that the cyclic disulphides formed within DTT are energetically favoured and more stable than noncyclic disulphide bonds [26]. However, by the same mechanism as for mucus glycoproteins, DTT could also affect the three-dimensional structure of membrane proteins. These effects could hamper immunological detection procedures based on specific antibodies and cause a decrease in antibody binding. As a consequence, fluorescence intensity, as measured by flow cytometry, could be severely altered.

Owing to the fact that the aim was to reveal the effect of DTT, cells that could be obtained without DTT pretreatment, with minimum likelihood for potential cell loss, had to be used. Other methods of mucus liquification, such as the use of a needle [11], repeated washing [12] or incubation with an enzyme mixture [13] might affect the inte- grity of cells and lead to selective recovery. Therefore, it was decided to use peripheral blood leukocytes that could be obtained with as few interventions as possible as a model for sputum cells. It has been suggested that the expression of surface markers, e.g. CD11b and CD18, is about three times higher in the peripheral blood of healthy donors as compared to sputum cells from patients with bronchiectasis [12]. The fact that, in the present data, the mean fluorescence was always $>8,000$ MESF and the limit of detection was $<1,000$ MESF renders it unlikely that, even with signals of half the intensity (as would be expected for induced sputum), the detection limit would be reached.

At least theoretically, the presence of mucus could affect immunological detection, e.g. by absorption of antibodies. However, the present data would remain valid if the concentration of DTT to which sputum cells are exposed were markedly reduced by interaction with mucins. Certainly some proviso is needed before extrapolating the present data to induced sputum. However, the arguments outlined above suggest that immunological staining for flow cytometry can be performed successfully in sputum cells which, before staining, have been necessarily separated from mucus.

The flow cytometric analysis of lymphocyte subsets in induced sputum is of particular interest. It requires antibodies directed against CD2, CD3, CD4, CD8, CD14, CD16, CD19, CD45, CD56 and HLA-DR. Until now, data referring to the effect of DTT on these markers have only been available in abstract form. The study reported that DTT did not alter the detection of CD3, CD4, CD8, CD14, CD19, CD25 and CD45 in blood leukocytes but led to a significant reduction in HLA-DR by $18 \%$ [14]. It remained unclear as to whether percentages of positive cells or fluorescence intensities had been evaluated. A later study which utilized flow cytometry and incubation with DTT showed that sputum lymphocytes differed between smokers and asthmatic subjects; however, this study did not investigate the effect of DTT [10].

Other data on the effect of DTT on immunological cell detection refer to surface markers which are not primarily important for the analysis of lymphocytes. As compared to samples prepared by repeated washing, treatment with $0.1 \%$ DTT caused a reduction in the mean fluorescence intensity of CD11b and CD18 in the sputum of patients with bronchiectasis and in the peripheral blood neutrophils of healthy donors [12]. In accordance with this, DTE caused a significant reduction in detectable levels of blood eosinophil CD11a, CD11b and CD18, whereas CD9, CD11c, CDw32 and CD35 were not affected [11].

As a result of these considerations, the effect of DTT on the flow cytometric detection of that panel of surface markers which is essential for lymphocyte subtyping was evaluated. To achieve optimal precision, its effect on the percentages of cells as well as on fluorescence intensity under strictly standardized conditions of calibration and quality control was assessed.

With the exception of CD14-positive monocytes and CD19-positive lymphocytes, the percentages of antibodypositive cells differed significantly between PBS- and DTT-treated samples. This was reflected in low Ri particularly for CD2, CD45 and HLA-DR (table 3). CD14 also showed a low $\mathrm{Ri}$, despite the fact that the difference between DTT- and PBS-treated samples was not significant. 
It should be noted, however, that, by its definition, the $\mathrm{Ri}$ does not contain all information regarding the comparison of DTT- and PBS-treated samples. As blood from subjects with normal white blood cell counts was investigated, the between-subjects variation was rather low as compared to the within-subjects variation, and that might have biased the Ri. Regarding the percentages of antibody-positive cells, duplicate samples, which were incubated separately, showed high Ri, thereby indicating high intra-assay reproducibility for most surface markers. The outliers may have occurred because the amount of antibody used for incubation was too small or because the vortex agitation of the sample was insufficient.

Regarding fluorescence intensity, all duplicate samples showed acceptable reproducibility. In CD2- and CD45-positive lymphocytes and CD14-positive monocytes, the fluorescence intensity was significantly reduced in DTT- as compared to PBS-treated samples. The authors suggest that epitopes are altered by DTT in such a way that the number of antibodies bound to the cell surface is reduced. Conversely, an increase in fluorescence intensity, as observed for CD16, might be due to the fact that changes in the adjoining structure expose epitopes. For HLA-DR, the present data differ from those of KIDNEY et al. [14] who found a reduction of $18 \%$. However, it is difficult to compare the results between studies as these authors did not give information about their method of data quantification.

Within the present data, the reduced fluorescence intensity of CD45- and CD2-positive lymphocytes was consistent with a lower percentage of lymphocytes, and the increased fluorescence intensity of CD16-positive granulocytes with a higher percentage of granulocytes. In contrast, it is difficult to reconcile the reduction in fluorescence intensity of CD14-positive monocytes after treatment with DTT, with the concomitant increase in the percentage of monocytes. In both cases, the effect of DTT on fluorescence intensity was small as reflected in high $\mathrm{Ri}$, with the exception of CD2 (table 3). It is noteworthy that the differences in fluorescence intensity between samples treated with DTT and PBS were not likely to be the cause of changes in the percentages of antibody-positive cells because positive and negative cells were always easily distinguishable.

The percentages of morphologically identifiable leukocytes differed slightly but significantly between PBS- and DTT-treated samples. The mechanism by which DTT caused these effects remains unclear; possibly they were linked to the lysis of erythrocytes which is not necessary for analysis of induced sputum. Ri for duplicate samples indicated higher reproducibility after DTT than after PBS. It should be noted, however, that the shifts in leukocyte numbers were well within the range of flow-cytometric accuracy.

The results of the present study were obtained in healthy subjects, and extrapolation to other groups, for example subjects with asthma, remains to be established. In addition, further investigations on the relationships between the state of activation and surface markers in leukocytes from blood and sputum, and on the influence of mucins would be helpful. Despite these limitations, however, the present study demonstrates that, with regard to percentages of antibody-positive cells, the effects of DTT are likely to be small.
In conclusion, dithiothreitol at the concentrations used in sputum processing affected some surface markers of peripheral blood leukocytes, with respect to both fluorescence intensity (CD2, CD14, CD16, CD45) and percentages of antibody-positive cells. However, the analysis suggests that dithiothreitol does not invalidate the comparison between different samples regarding the immunocytochemical analysis of lymphocyte surface markers.

Acknowledgements. The work was supported by the LVA - Freie und Hansestadt Hamburg, Germany, and the Labor Dr. Kramer und Kollegen, Geesthacht, Germany. The authors would like to thank O. Holz for helpful comments on the manuscript.

\section{References}

1. Pin I, Gibson PG, Kolendowicz R, et al. Use of induced sputum cell counts to investigate airway inflammation in asthma. Thorax 1992; 47: 25-29.

2. Fahy JV, Liu J, Wong H, Boushey HA. Cellular and biochemical analysis of induced sputum from asthmatic and from healthy subjects. Am Rev Respir Dis 1993; 147: 1126-1131.

3. Takahashi M. Respirationstrakt. Farbatlas der onkologischen Zytologie. Erlangen, Fachbuch-Verlagsgesellschaft, 1987; pp. 267-270.

4. Pavord ID, Pizzichini MM, Pizzichini E, Hargreave FE. The use of induced sputum to investigate airway inflammation. Thorax 1997; 52: 498-501.

5. Tockman MS, Qiao Y, Li L, et al. Safe separation of sputum cells from mucoid glycoprotein. Acta Cytol 1995; 39: 1128-1136.

6. Keatings VM, Evans DJ, O'Connor BJ, Barnes PJ. Cellular profiles in asthmatic airways: a comparison of induced sputum, bronchial washings, and bronchoalveolar lavage fluid. Thorax 1997; 52: 372-374.

7. Hargreave FE, Pizzichini MM, Pizzichini E. Assessment of airway inflammation. In: Barnes PJ, Grunstein MM, Leff AR, Woolcock AJ, eds. Asthma, Philadelphia, Pa, Lippincott-Raven, 1997; pp. 1433-1449.

8. Gelder CM, Thomas PS, Yates DH, Adcock IM, Morrison JF, Barnes PJ. Cytokine expression in normal, atopic, and asthmatic subjects using the combination of sputum induction and the polymerase chain reaction. Thorax 1995; 50: $1033-1037$.

9. Holz O, Richter K, Jörres RA, Speckin P, Mücke M, Magnussen $H$. Changes in sputum composition between two inductions performed on consecutive days. Thorax 1998; 53: 83-86.

10. Kidney JC, Wong AG, Efthimiadis A, et al. Elevated B cells in sputum of asthmatics. Close correlation with eosinophils. Am J Respir Crit Care Med 1996; 153: 540544.

11. Hansel TT, Braunstein JB, Walker C, et al. Sputum eosinophils from asthmatics express ICAM-1 and HLADR. Clin Exp Immunol 1991; 86: 271-277.

12. Wu MS, Hwang KH, Lin HC, Kuo HP. The preservation of neutrophil activity in induced sputum by repeated washing and dithiothreitol method. Am J Respir Crit Care Med 1997; 155: A622.

13. Cai Y, Carty K, Gibson P, Henry R. Comparison of 
sputum processing techniques in cystic fibrosis. Pediatr Pulmonol 1996; 22: 402-407.

14. Kidney JC, Efthimiadis A, Incretolli V, Kolendowicz R, Dolovich J, Hargreave FE. Evaluation of lymphocyte subclasses in sputum. J Allergy Clin Immunol 1994; 93: 168.

15. Louis R, Shute J, Goldring K, et al. The effect of processing on inflammatory markers in induced sputum. Eur Respir J 1999; 13: 660-667.

16. Kidney JC, Wong AG, Efthimiadis A, et al. Sputum lymphocyte subclasses and activation measured by flow cytometry. Am J Respir Crit Care Med 1994; 149: A572.

17. Loppow D, Böttcher M, Jörres RA, Gercken G, Magnussen H. Flow cytometric analysis of induced sputum lymphocyte subpopulations. Am J Respir Crit Care Med 1999; 159: A514.

18. Böttcher M, Abel G. Immunophänotypisierung von Lymphozyten in der Bronchoalveolären Lavage. Krefeld, Coulter Electronics, 1994.

19. Holz O, Jörres RA, Koschyk S, Speckin P, Welker L, Magnussen $\mathrm{H}$. Changes in sputum composition during sputum induction in healthy and asthmatic subjects. Clin Exp Allergy 1998; 28: 284-292.
20. Richter K, Holz O, Jörres RA, Mücke M, Magnussen H. Sequentially induced sputum in patients with asthma or chronic obstructive pulmonary disease. Eur Respir $J$ 1999; 14: 697-701.

21. Lopez-Vidriero MT, Reid L. Bronchial mucus in health and disease. Br Med Bull 1978; 34: 63-74.

22. Liu YC, Khawaja AM, Roger DF. Pathophysiology of airway mucus secretion in asthma. In: Barnes PJ, Rodger IW, Thompson NC, eds. Asthma: Basic Mechanisms and Clinical Management, 3rd edn. San Diego, CA, Academic Press, 1998; pp. 205-227.

23. Lopez-Vidriero MT, Reid L. Respiratory tract fluid chemical and physical properties of airway mucus. Eur $J$ Respir Dis 1980; 110: 21-26.

24. Lundgren JD, Shelhamer JH. Pathogenesis of airway mucus hypersecretion. J Allergy Clin Immunol 1990; 85: 399-417.

25. Popov T, Gottschalk R, Kolendowicz R, Dolovich J, Powers P, Hargreave FE. The evaluation of a cell dispersion method of sputum examination. Clin Exp Allergy 1994; 24: 778-783.

26. Bodanszky M. Peptide Chemistry. 2nd Edn. Berlin, Springer Verlag, 1993; pp. 34-38. 